\title{
El superlativo absoluto en el español peninsular del siglo XX
}

\author{
The absolute superlative in 2oth century \\ Peninsular Spanish
}

\author{
VÍCTOR LARA BERMEJO \\ Institut für spanische Sprache und Literaturen \\ Universität Bern \\ Länggassstrasse 49. CH-3000 Bern 9. Suiza \\ victor.lara@rom.unibe.ch \\ Orcid ID 0000-0002-1068-8553
}

Resumen: El superlativo absoluto en español puede construirse mediante el adverbio muy o el sufijo-ísimo. A pesar de que la reintroducción de la forma morfológica ha sido datada de finales de la Edad Media, investigaciones más recientes apuntan al siglo XIX como fecha clave para su popularización. Con el fin de analizar su distribución dialectal en el español peninsular a lo largo del siglo XX, hemos extraído datos del Atlas lingüístico de la Península Ibérica (ALPI) y de otros posteriores. De acuerdo con estos, observamos que, efectivamente, el sufijo -ísimo está circunscrito a un área geográfica determinada, mientras que muy ocupa la zona restante, sin dar apenas ocurrencias de la solución morfológica. A ello hay que añadir el uso de mucho (muncho en ciertos enclaves) como grado del adjetivo, siendo este fenómeno una reminiscencia del estado lingüístico del paso del latín al español.

Palabras clave: Dialectología. Geolingüística. Adverbios. Superlativo. -ísimo.
RECIBIDO:7 DE ENERO DE 2016 ACEPTADO: 19 DE ABRIL DE 2016

\begin{abstract}
The absolute superlative in Spanish can be expressed with the adverb muy or the suffix -ísimo. Despite the fact that the reintroduction of the morphological strategy is said to have occurred in the late Middle Ages, latest research point out that the 19th century was crucial for its universalisation. With the aim of analysing its dialect distribution in Peninsular Spanish throughout the 2oth century, I have collected data from the Linguistic Atlas of the Iberian Peninsula (ALPI) and other carried out later. Based on these results, the suffix -isimo is restricted within a specific geographic area whereas muy occupies the rest of the studied zone. Furthermore, some dialects still use mucho (muncho in certain municipalities) to produce the degree of the adjective, the same way they did it in the evolution from Latin to Spanish.
\end{abstract}

Keywords: Dialectology. Geolinguistics. Adverbs. Superlative. -ísimo. 
EL SUPERLATIVO: DEL LATÍN AL ESPAÑOL

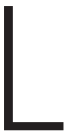
a expresión de la superlación en latín solía hacerse mediante la estrategia morfológica -ISSIMUS-A-UM, si bien llegó a convivir con consPLURIMUM más adjetivo (Rohlfs; Bruyne 1980 y 1986; Pinkster; Ledgeway). La principal diferencia entre las dos estrategias residía en que la sintética servía para la superlación absoluta, mientras que la analítica era más productiva para la superlación relativa. A todas estas construcciones, hay que añadir la compuesta por el indefinido MULTUS o el adverbio BENE más adjetivo como alternativa para el superlativo absoluto (Sidwell).

El paso a las lenguas romances provocó el auge de las construcciones analíticas con MULTUS (mucho en español), las cuales no volvieron a recuperar la forma sintética hasta la moda italiana del siglo XVI. No obstante, en el caso del español, MULTUS se dividió en dos. Por un lado, se palatalizó de la siguiente manera: MULTUS $>$ muito $>$ mucho $>$ much; por otro, produjo el mismo diptongo que se desarrolló en portugués: MULTUS $>$ muito $>$ muit $>$ mui / muy (Alvar/Pottier). Para Coromines y Pascual o Serradilla (2005), mucho coexistía con muito en el castellano preliterario. Es más, de acuerdo con estos autores, la anteposición del indefinido con respecto al adjetivo provocaba la caída de la $o$ final (muit) y, en este caso, si el adjetivo empezaba por consonante, la $t$ no se palatalizaba, por lo que acabó por evolucionar en muy (muy bueno). Es el inicio en vocal por parte del elemento cuantificado lo que facilitaba la pronunciación de la opción palatalizadora mucho, con caída de la vocal final en la época de la apócope extrema (much alto). Una vez transcurrida la época afrancesada de la apócope extrema, se encuentran más ocurrencias de mucho alto y se agrava la vacilación entre mucho y muy, cuya oscilación permanece con gran productividad hasta finales del siglo XV. Para Camus (2006 y 2008), es en el siglo XVII cuando se fijan los valores de muy (y tan) y mucho (y tanto). El primero intensifica el adjetivo o el adverbio, mientras que mucho se utiliza para sintagmas de medida y frases adverbiales. Antes de esa fecha, muy y mucho se alternaban, incluso seguidos de más, menos, mejor o peor.

La diferenciación entre el grado del adjetivo y la cuantificación indefinida (conceptos estrechamente ligados para Camus 2006) puede hacerse mediante dos estrategias: el uso de un mismo indefinido (variable para el caso de la cuantificación e invariable para el grado del adjetivo) o la distinción entre dos formas diversas (una que funcione como un adjetivo indefinido y otra que 
lo haga como un adverbio de grado). Mientras que el primer caso se atestigua en la mayoría de las lenguas romances o el griego (molt en catalán, molto en italiano, muito en portugués, тo $\lambda u ́$ en griego, e incluso en zonas del español peninsular como La Rioja, Navarra, Aragón o áreas de Andalucía oriental, de acuerdo con Llorente), el segundo se manifiesta en muchas otras. Por ejemplo, el inglés posee very como adverbio para el superlativo absoluto y much I many como adjetivo indefinido cuantificador. La forma very proviene del francés vrai ('verdadero') y se introduce en la lengua común en época medieval; la especialización de much (también de época medieval) es una herencia de mickle ('gran cantidad'), de origen germánico. Asimismo, como en español, la unión con los adverbios more y less ('más' y 'menos') induce la expresión de much en lugar de very (Lass y otros). El alemán también se caracteriza por un comportamiento similar, puesto que distingue entre sehr ('muy') y viel ('mucho'), aunque el primero puede cuantificar una acción (imposible en español, aunque no con mucho), como en Ich arbeite sebr ('Trabajo mucho') (Keller); el inglés opta por la concatenación de very más el cuantificador para estos casos: I work very much ('Trabajo mucho'). En el caso del francés, el heredero moult funcionaba como el resto de sus homólogos romances (variable e invariable), pero decayó en el siglo XVI a expensas de la lexicalización de beaucoup. Sin embargo, antes de la victoria de beaucoup, el adverbio très ya funcionaba como marca de superlativo en el siglo XI. La etimología de este último proviene de TRANS ('más allá de') y podía equivaler a jusqu'à ('hasta' o 'completamente') (Centre national de la recherche scientifique).

Volviendo al español, el adverbio bien como intensificador y sinónimo de muy, así como la opción epentética de muncho, han dado lugar a un debate sobre su uso a lo largo de la historia. Así, Camus (2006 y 2008) y Serradilla (2007) sostienen que el uso común de bien y muy como intensificadores se fija en el siglo XVII, mientras que muncho está presente en el español hasta el siglo XVI, conservándose hoy día en el registro oral de ciertas zonas dialectales (tesis que ya apuntaban Coromines y Pascual). Para Serradilla (2004 y 2008), el adverbio muy fue la estrategia más usada a lo largo de la historia del español para construir el grado superlativo absoluto, en detrimento de bien u otras construcciones como -ísimo. A este respecto, Zieliński opina que-ísimo fue resultado de una koiné eclesiástica con predominio latinizante, que se fue extendiendo con la moda italiana tras el Descubrimiento de América. Sus resultados constatan que no era tan universal como se había supuesto y a finales del XVIII ya empieza a ser común. 


\section{LA EVOLUCIÓN GEOLINGÜÍSTICA DEL SUPERLATIVO EN EL SIGLO XX}

La primera herramienta dialectal que utilizaremos para cartografiar la situación del superlativo absoluto es el Atlas linguiístico de la Península Ibérica (ALPI). Dicho trabajo data de mediados del siglo XX y pretendía dar cuenta de la variación léxica, morfosintáctica y fonética de las lenguas romances en todo el territorio peninsular. Este atlas dialectal fue creado por Tomás Navarro Tomás y llevado a cabo por tres equipos repartidos por la zona castellana, la gallego-portuguesa y la catalano-valenciana, con dos investigadores nativos en cada grupo. Se encuestaron 527 enclaves rurales y en cada uno de ellos se entrevistó a un informante con pocos niveles de estudio, que rondara entre los cuarenta y sesenta años y que toda su vida hubiera vivido en el pueblo. Las entrevistas fueron realizadas entre los años 1920 y 1950 y consistían en oraciones y palabras preconcebidas que los informantes repetían en su variedad vernácula y cuyos resultados eran manuscritos fonéticamente. La metodología puede plantear dudas acerca de la espontaneidad de las respuestas del informante, así como de los escasos datos sobre los fenómenos vernáculos, ya que tan solo se recogía una respuesta por pregunta y se encuestaba a un solo informante por enclave.

Dentro de estas oraciones, hay una única pregunta donde se incluya el adverbio muy: Un café muy fuerte (pregunta $\mathrm{F}$ del cuaderno I). Será esta la que analizaremos como punto de partida, de acuerdo con el mapa 1.

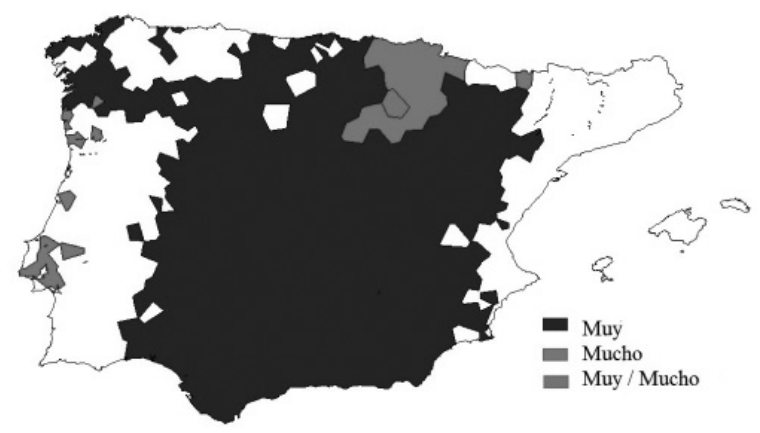

Mapa 1. Un café muy fuerte

La pregunta preestablecida por el ALPI que se cartografía en el mapa 1 muestra una preponderancia de muy en todo el español peninsular, excepto en zo- 
nas centro-orientales del norte. Es en la totalidad de Navarra, la zona de La Rioja colindante con la primera, la mitad norte de Soria, Álava y zonas septentrionales de Huesca (con la forma muto, ya documentada en el paso del latín al romance en zona aragonesa por Coromines y Pascual), donde se ha optado por la solución un café mucho fuerte, conservando el estadio medieval al que nos referíamos al principio. En el resto de las lenguas romances peninsulares no se ha recogido la respuesta, salvo en zonas del portugués, donde la forma muito es la elegida.

Hay que advertir de que la metodología del ALPI no es la idónea para registrar datos cuantitativos, pero nos sirve de punto de partida y de referencia para fenómenos lingüísticos vigentes en el siglo XX. Aunque tan solo se obtuvo una respuesta por pregunta y municipio, muchos de sus hallazgos se han confirmado posteriormente, por lo que no cabe desdeñar dicho corpus. En cualquier caso, de acuerdo con los resultados, la confusión entre muy y mucho aún se conservaba hace 75 años en las zonas anteriormente mencionadas, a pesar de que la Real Academia Española afirma que mucho para el grado del adjetivo desapareció hace mucho tiempo (NGLE).

Si detallamos algo más las respuestas, encontramos varias fórmulas dentro de la opción canónica muy, como se observa en el mapa 2.

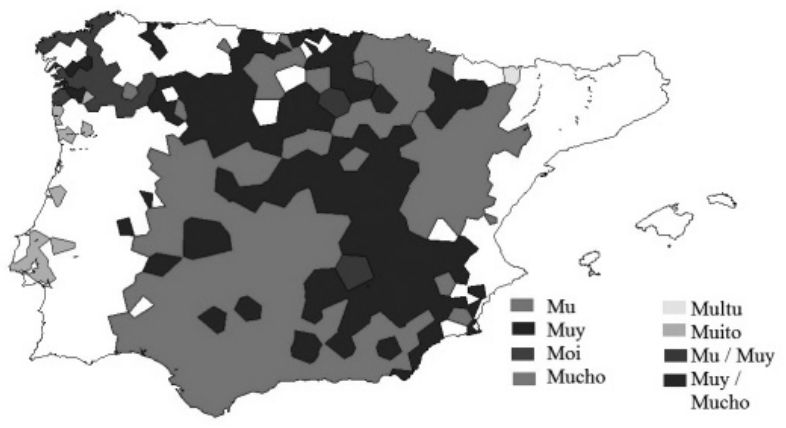

Mapa 2. Variantes de MULTUS

Hemos hallado ocho soluciones distintas, sin discriminación de lengua. Así, la opción mayoritaria que se extiende por el centro-occidente, toda Andalucía y Aragón es mu. La segunda opción, la forma plena muy, se esparce por el castellano oriental (sobre todo, Castilla-La Mancha, Murcia, el este de Castilla y León), zonas centrales como Madrid, Cantabria, León y Zamora, y de manera esporádica en áreas occidentales. El gallego prefiere moi; el portugués, muito; 
la forma mucho se da en las zonas descritas en el mapa 1; y observamos un caso en Huesca de multu y oscilaciones entre muy y mucho en Navarra, y $m u$ con muy en enclaves aislados.

Para Givón, los adjetivos son muy propensos a variación lingüística, en especial, si hacen referencia a cualidades abstractas o se comportan como modificadores funcionales del sustantivo (deícticos, indefinidos, numerales, etc.). Dicha variación, en el caso que nos ocupa, se muestra en la reducción fónica de mucho a favor de muy, cuyo funcionamiento se ha especializado para expresar el grado del adjetivo. Este hecho parece relacionarse con un proceso de gramaticalización, puesto que una palabra funcional (como es el indefinido) ha visto reducido su peso fónico y se ha reinterpretado como otra palabra funcional, en este caso, reiteramos, como grado superlativo absoluto del adjetivo. De acuerdo con las afirmaciones de Hopper y Traugott, las vías de gramaticalización no solo surgen en el paso de una palabra léxica a una funcional, sino en la reinterpretación de una funcional a otra de la misma categoría. Asimismo, cabe destacar que, de acuerdo con el mapa 2, muy sigue sufriendo una reducción de su peso fónico; prueba de ello es la solución $m u$, extendida en el sur y centro peninsulares, calificada de vulgar.

En principio, tal y como acontece en las demás lenguas romances peninsulares, el uso de mucho como superlativo es invariable (puesto que se trata de un adverbio y no de un adjetivo), es decir, no se rige por el número y género del adjetivo al que modifica. El trabajo de Pato y Viejo, quienes hacen una búsqueda de mucho como superlativo en el español peninsular actual, refleja efectivamente la invariabilidad del cuantificador en este tipo de construcciones. De todos los ejemplos sacados del Corpus Oral y Sonoro del Español Rural (COSER), basado en la entrevista semidirigida, ninguno concierta en género y número con el referente, y todos los casos se circunscriben a las zonas de La Rioja, Soria, Guadalajara, Navarra, todo Aragón y partes de Murcia y Almería. Aunque tanta difusión no es la que reflejan los datos de hace un siglo (recordemos los problemas metodológicos que plantea el ALPI), los autores recuerdan que el uso de mucho viene condicionado por el factor edad; es decir, son los hablantes más ancianos, de ámbito rural y sin estudios los que producen este fenómeno. Asimismo, en todos los enclaves, el uso de mucho oscilaba con el de muy, por lo que la opción estándar parece pugnar actualmente por establecerse.

Como hemos mencionado, el superlativo absoluto podía construirse en latín de una manera sintética con los sufijos-ISSIMUS, -ILLIMUS o-ERRIMUS o analítica. Tras analizar las construcciones analíticas recogidas en el ALPI, el 
cuestionario previó dos oraciones con un superlativo morfológico: la pregunta 277 Una moza guapísima y la 278 Una mazorca grandísima. A continuación, ilustramos los resultados en los mapas 3 y 4.

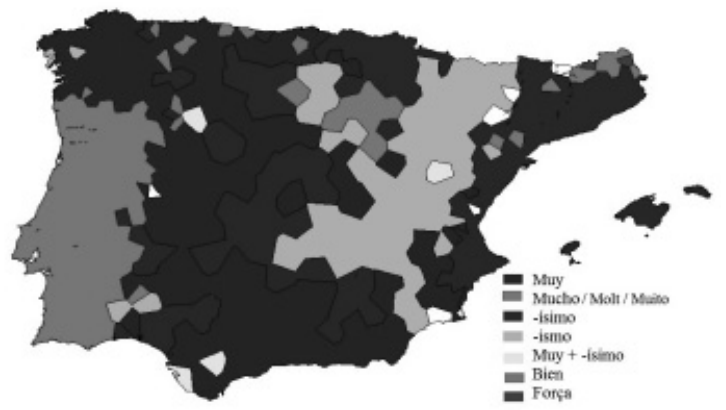

Mapa 3. Formas de superlativo de Una moza guapísima

De acuerdo con el mapa 3, la zona centro oriental de la Península Ibérica opta por el cultismo -ísimo mientras la centro occidental se mantiene con la estrategia perifrástica muy o mucho, salvo algunos enclaves esporádicos. De nuevo, mucho se atestigua en Portugal (muito) y en zonas de Soria, mientras que bien se documenta en la zona asturiana y leonesa, como ya indicaban Serradilla (2005, 2006 y 2008) o Gómez Torrego. En el catalán, hemos hallado força como intensificador en enclaves aislados del área francesa y molt en la parte más septentrional. En el resto del territorio peninsular, observamos una reduplicación del superlativo en puntos del este y el sur, con respuestas como Una moza muy guapísima. En la región donde predomina la forma morfológica, existen dos soluciones: -ísimo e-ismo.

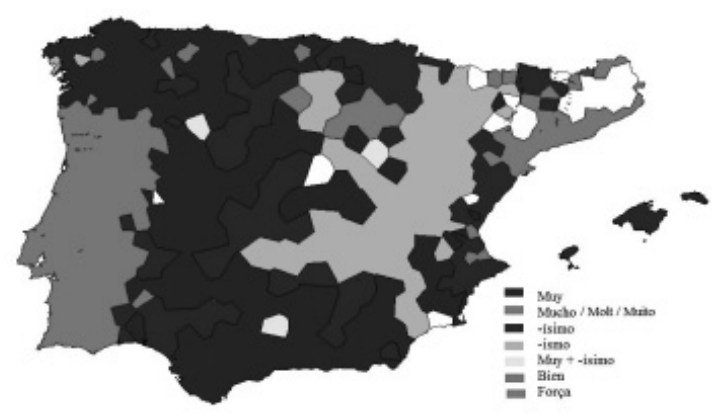

Mapa 4. Formas de superlativo de Una mazorca grandísima 
El mapa 4 no difiere mucho del número 3, con la excepción de que la zona catalana ha optado mayoritariamente por molt en lugar del sufijo superlativo. La confrontación entre el área con la construcción perifrástica y la sintética sigue siendo prácticamente la misma, como lo es la pérdida del acento proparoxítono en el este peninsular. Asimismo, dos enclaves han producido una doble superlación: muy grandísima. A este respecto, se han referido distintos autores (Palomo; Serradilla 2009; Pons), quienes admiten que la doble superlación ya se daba en el español clásico y fue perdiendo aceptación social, sobre todo por parte de los gramáticos, hacia el siglo XVIII. Para Sánchez López (1999 y 2006) y Sáez del Álamo, no obstante, la doble superlación, en principio, no puede manifestarse.

Cabe preguntarse varias cuestiones: ¿por qué la zona occidental ha descartado la opción culta? ¿Qué extensión posee actualmente la caída de la esdrújula en el oriente? Con relación a la primera pregunta, surge la duda sobre la metodología empleada y cómo indujeron la respuesta a ambas oraciones, ya que todo el occidente ha sido coherente. En una primera visión, se tiene la tentación de afirmar que el cultismo en -ísimo, de procedencia italiana a partir del siglo XVI, a pesar del paso del tiempo, no estaba tan arraigado en la lengua más vernácula de mediados de la centuria pasada. No obstante, el cambio de respuesta ante un mismo tipo de pregunta por parte de los hablantes catalanes refuerza la duda acerca del planteamiento metodológico. A pesar de ello, Azofra sostiene que -ísimo se introduce en el español para términos religiosos y no empieza a ser popular en el habla popular hasta finales del siglo XIX, tesis apoyada por Wang. La bibliografía consultada admite que el superlativo morfológico sufre una aceptación desigual, según la época, siendo un préstamo culto o semiculto. Por tanto, de acuerdo con sus observaciones y las investigaciones de Azofra y Wang, los datos que ilustran los mapas 3 y 4 pueden perfectamente ser reflejo de la realidad del momento de las encuestas. De ser así, la generalización de -ísimo en la lengua popular estaría condicionada, al menos, por un factor geográfico; su introducción se da en un ámbito oriental (Baleares, Cataluña, Comunidad Valenciana, parte de Aragón y Castilla-La Mancha) (Moreno Fernández) y pugna con la opción analítica (muy / mucho / muito) en el centro y occidente (a excepción de las zonas portuguesa y gallega). Si comparamos estos datos con atlas posteriores, obtenemos los resultados que se cartografían en el mapa 5 . 


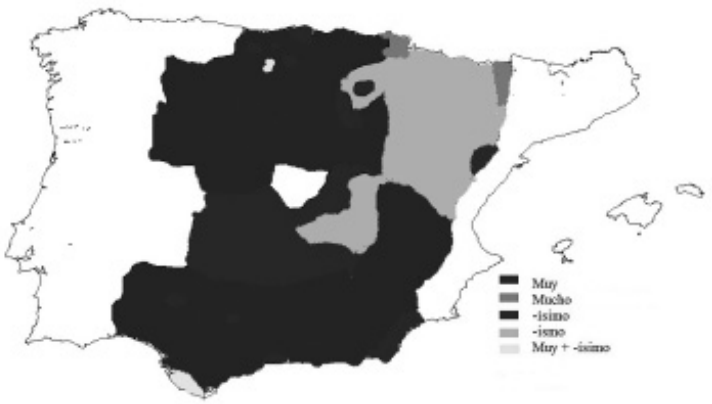

Mapa 5. Formas de superlativo en los atlas lingüísticos posteriores al ALPI

Así, observamos que, por ejemplo en el caso del ALECMAN, todo Castilla-La Mancha produce el sufijo -ísimo, por lo que a finales del siglo XX, la opción sintética estaba plenamente establecida en el habla vernácula de dicha zona. En el caso del Atlas linguiistico y etnográfico de Cantabria (ALECANT), se muestra que los hablantes optan por muy en lugar del sufijo -ísimo (muy esporádico) en la pregunta Una espiga grandísima. Asimismo, la doble superlación tan solo se halla en un enclave del sur (Una espiga muy grandísima). Por su parte, el Atlas linguiístico y etnográfico de Andalucía (ALEA) cartografía las frases Una mazorca grandísima y Una moza guapísima. En ambas, el porcentaje de mu(y) es mayor que la opción sintética, si bien la segunda oración presenta mayores ocurrencias de muy y una proporción baja de -ísimo, sin que haya un patrón geográfico determinado. Se documenta la doble graduación en el suroeste de la provincia de Cádiz. El Atlas lingüístico y etnográfico de Aragón, Rioja y Navarra (ALEANR) proporciona información de las frases Una muchacha guapísima y Una espiga grandísima. De acuerdo con este trabajo, el uso de la estrategia analítica se circunscribe al extremo oriental de Aragón (mot) y al extremo noroccidental de Navarra (mucho). El resto de la zona encuestada muestra la solución-ísimo.

Por último, el Atlas lingüístico de Castilla y León (ALCYL) preestablece la oración Una muchacha guapísima y muestra que la estrategia mu(y) es la solución abrumadora en todo el territorio, con esporádicas respuestas de -ísimo. Asimismo, el uso de mucho como superlativo se ha recogido en el este de Burgos, la mitad oriental de Soria y, de manera esporádica, en zonas occidentales de dicha provincia. 
Con respecto a la segunda de las cuestiones, González Ollé, Pastor Blanco o Buesa puntualizan que las variedades orientales tienden a deshacer la acentuación esdrújula favoreciendo la paroxítona. De hecho, palabras como sábana, bígado o nombres propios como Ángeles se producen como sabána, higádo, Angéles. La solución en -ismo tan solo sería una forma más de hacer paroxítona la construcción superlativa proparoxítona. En el mapa 5 se aprecian todos los datos de los atlas lingüísticos que ofrecen una pregunta a este respecto. El ALECMAN ofrece una pregunta (una mazorca grandísima) a partir de la cual podemos comparar el desarrollo de -is(i)mo en la centuria anterior. De acuerdo con esta, la solución paroxítona se manifiesta en Guadalajara (excepto el extremo oeste), en Cuenca y Albacete (salvo algún enclave aislado) y en el centro oriente de Ciudad Real, siendo inexistente en Toledo. En el ALEA, no se encuentran ocurrencias de -ismo en Andalucía oriental, en contra de los datos que exponen Pato y Viejo en su investigación. En el caso del ALEANR, -ismo se extiende por toda la Rioja, Navarra (salvo cierta área septentrional) y la mayor parte de Aragón, excepto el extremo meridional. Por último, el ALCYL muestra un área muy acotada del este de Palencia, donde se opta por $-i s m o$.

Aunque hemos obtenido muy pocos ejemplos de la doble graduación, Jörnving opina que, aunque la introducción de -isimo convivió con muy y otras fórmulas de superlativo, no desbancó a muy al principio, por lo que era relativamente usual la coocurrencia de ambas estrategias. De hecho, hay muchos autores que previenen de la no sinonimia plena entre muy e -ísimo, por lo que es posible su coexistencia para modificar un mismo adjetivo dentro de una oración determinada (Galeote). Esta diferencia se aprecia en dos contextos: por un lado, hoy día se pueden escuchar construcciones como No está muy lejos, está lejísimos, No es muy guapo, es guapísimo; por otro, mientras -ísimo no puede, por ejemplo, hacer un superlativo de adjetivos helenos (*demagoguísimo), muy no presenta restricciones (muy demagogo) (Sánchez López 2006).

Por último, aunque ya se ha comentado anteriormente, nos gustaría mostrar la cartografía de los resultados que obtuvieron Pato y Viejo, quienes, aunque no se detuvieron en las formas sintéticas, han dado cuenta de la vigencia de mucho como adverbio de superlación (mapa 6). 


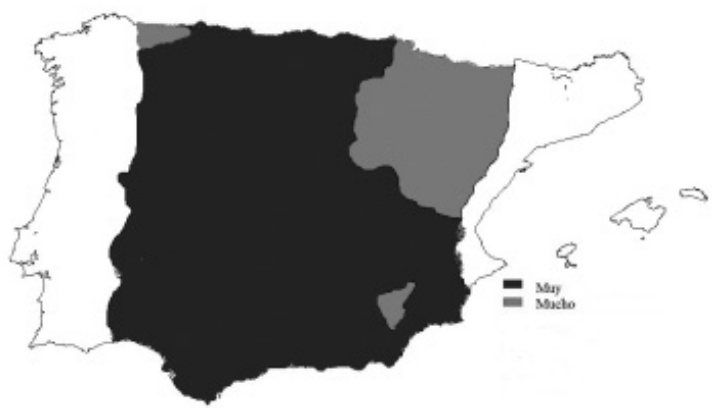

Mapa 6. Uso de mucho / muy como grado del superlativo absoluto (COSER)

Como se observa en el mapa 6, basado en los datos del Corpus Oral y Sonoro del Español Rural (COSER), el uso de mucho surge en zonas del suroriente peninsular, el occidente de Asturias y se esparce, siempre de acuerdo con Pato y Viejo, por toda Navarra, La Rioja, Aragón, Soria y Guadalajara. Sin embargo, a pesar de los datos de los atlas lingüísticos analizados, en el caso del COSER, el uso de mucho convive con el estándar muy.

Si resumimos todos los resultados de todas las herramientas que hemos manejado, observamos que, efectivamente, el uso de mucho como adverbio de grado en español es de tipo oriental, es decir, se da en la zona más al este del área castellana. Las pruebas indican que se ha dado en todo el siglo pasado con mucha fuerza en Navarra, La Rioja y las zonas colindantes. No obstante, las preguntas formuladas en los atlas lingüísticos se reducían a la obtención de la estrategia sintética (-ísimo) (excepto el ALPI, que sí preestablecía una frase con muy) y recogían una respuesta por enclave. Esa carencia cuantitativa ha podido influir para que el empleo de mucho en lugar de muy no surgiera en toda la zona que da el COSER, cuya metodología proporciona mayores ocurrencias. En cualquier caso, parece claro que muy se va imponiendo en el ámbito más vernáculo del español peninsular, así como la estrategia sintética en -ísimo, cuya extensión no era tan prolífica en la primera mitad del siglo XX.

\section{CONSIDERACIONES FINALES}

Los datos de que disponemos muestran que el empleo de mucho como superlativo se daba a mediados del siglo XX en zonas del norte peninsular, en concreto, en Navarra, Álava, La Rioja, Soria y parte de Huesca en la variedad lingüística aragonesa. Su vigencia se ha mantenido con menor fuerza, según 
los atlas llevados a cabo en la segunda mitad de la centuria pasada, y parece mantenerse, aunque en hablantes con un perfil muy determinado, en las mismas zonas y en enclaves del sureste peninsular (si bien varía con la opción estándar muy). Asimismo, hemos observado la invariabilidad de la forma, tal y como ocurre en las demás lenguas peninsulares y en italiano. En la zona del país donde se diferencia entre mucho y muy, advertimos una evolución ulterior en la gramaticalización de mucho a muy, puesto que el peso fónico se ha reducido algo más, sobre todo en el sur y centro de la península, dando como resultado $m u$, tachado de vulgar por muchos autores.

La comparación de distintas fuentes geolingüísticas del siglo XX ha posibilitado trazar la evolución del superlativo absoluto. Además del uso de mucho, hemos visto que la opción sintética (-ísimo) no parecía ser general en el habla vernácula de la primera mitad del siglo pasado. Su extensión se circunscribía al oriente peninsular y alternaba con muy / muito con menor fuerza cuanto más al occidente nos desplazásemos. Dentro de esta área, la parte más al este eliminaba la acentuación proparoxítona por la paroxítona, produciendo -ismo. Los atlas lingüísticos posteriores confirman en parte esta tendencia, puesto que -ísimo es más prolífico en zonas orientales de las áreas encuestadas, aunque aún convive con muy en toda Andalucía, si bien el adverbio sigue siendo predominante.

Asimismo, la evolución sintáctica de mucho en el español es desigual. En zonas norteñas, se mantiene en un estadio primitivo antes de su disociación entre grado y cuantificador, el mismo estadio que aún poseen el portugués, el catalán, el griego o el italiano. En el resto del territorio peninsular, la especialización de la forma de grado no se ha completado totalmente y se mantiene como la situación del inglés, donde el grado no puede intensificar un verbo. Así, el inglés recurre al cuantificador much precedido del adverbio de grado very, mientras que el español opta por el cuantificador mucho, aunque a veces puede verse modificado por muy (Me gusta muy mucho). La fase ulterior sería la que presenta el alemán, donde sebr (el adverbio de grado) puede emplearse también para expresar el grado de una acción.

\section{OBRAS CITADAS}

ALCYL: Manuel Alvar. Atlas lingüístico de Castilla y León. Valladolid: Junta de Castilla y León/Fundación Duques de Soria, 1999. 
ALEA: Manuel Alvar y otros. Atlas lingüistico y etnográfico de Andalucía. Granada: Universidad de Granada/Madrid: CSIC, 1961-1973.

ALEANR: Manuel Alvar. Atlas lingüístico y etnográfico de Aragón, Navarra y Rioja. Zaragoza: Diputación Provincial de Zaragoza/Madrid: CSIC, 1979-1983.

ALECANT: Manuel Alvar. Atlas lingüístico y etnográfico de Cantabria. Madrid: Arco Libros, 1995.

aleCMAn: Pilar García Mouton y Francisco Moreno Fernández. Atlas lingüístico (y etnográfico) de Castilla-La Mancha. Alcalá de Henares: Universidad de Alcalá, 1988-1994.

ALPI: David Heap. Atlas lingüístico de la Península Ibérica. Ontario: University of Western, 2003.

Alvar, Manuel, y Bernard Pottier. Morfología histórica del español. Madrid: Gredos, 1987.

Azofra Sierra, María Elena. Morfosintaxis histórica del español: de la teoría a la práctica. Madrid: Universidad Nacional de Educación a Distancia, 2009.

Bruyne, Jacques. "Acerca de la traducción de -ísimo". LEA 2.1 (1980): 27-37.

Bruyne, Jacques. "Onomástica y elativos en -ísimo". Anuario de Lingüística Hispánica 2 (1986): 9-20.

Buesa Oliver, Tomás. "Particularidades del español hablado en Aragón". Fornadas de Filología Aragonesa: en el L aniversario del AFA. Ed. José María Enguita. Zaragoza: Institución Fernando el Católico, 1999. 113-38.

Camus, Bruno. "Cuantificadores I: los cuantificadores propios". Sintaxis histórica de la lengua española, II: La frase nominal. Dir. Concepción Company Company. México D.F.: Fondo de Cultura Económica/UnAM, 2006. 881-962.

Camus, Bruno. "De adjetivos a cuantificadores: un fragmento de la historia de los indefinidos románicos". Actas del XXXVII Simposio Internacional de la Sociedad Española de Linguística. Eds. Inés Olza Moreno y otros. Pamplona: Servicio de Publicaciones de la Universidad de Navarra, 2008. 87-95.

Centre national de la recherche scientifique. Ortolang. Nancy: Université de Nancy/CNRS/CNRT, 2005.

Coromines, Joan, y José Antonio Pascual. Diccionario crítico etimológico castellano e hispánico. Madrid: Gredos, 1980-1991.

COSER: Inés Fernández-Ordóñez. Corpus Oral y Sonoro del Español Rural. Madrid: Universidad Autónoma de Madrid, 1989-2016.

Galeote, Manuel. El habla rural del Treviño de Iznájar, Villanueva de Tapia y Venta de Santa Bárbara. Granada: TAT, 1988. 
Givón, Talmy. Syntax: An Introduction. Amsterdam/Philadelphia: John Benjamins, 2001.

Gómez Torrego, Leonardo. Gramática didáctica del español. Madrid: SM, 2000. González Ollé, Fernando. El babla de la Bureba: introducción al castellano actual de Burgos. Madrid: CSIC/Patronato Menéndez Pelayo/Instituto Miguel de Cervantes, 1964.

Hopper, Paul, y Elizabeth Closs Traugott. Grammaticalization. Cambridge: Cambridge UP, 2003.

Jörnving, Rolf. "El elativo en -ísimo en la lengua castellana de los siglos XV y XVI”. Studia Neophilologica 34.1 (1962): 57-85.

Keller, Rudolf Ernst. The German Language. Copenhague: Humanities Press International, 1978.

Lass, Roger, y otros. The Cambridge History of the English Language. 6 vols. Cambridge: Cambridge UP, 1992-2001.

Ledgeway, Adam. From Latin to Romance: Morphosyntactic Typology and Change. Oxford: Oxford UP, 2012.

Llorente, Antonio. "Algunas características lingüísticas de La Rioja en el marco de las hablas del valle del Ebro y de las comarcas vecinas de Castilla y Vasconia”. Revista de Filología Española 48 (1965): 287-315.

Moreno Fernández, Francisco. "Castilla la Nueva". Manual de dialectología hispánica: el español de España. Dir. Manuel Alvar. Barcelona: Ariel, 1996. 213-32.

NGLE: Real Academia Española, y Asociación de Academias de la Lengua Española. Nueva gramática de la lengua española. Madrid: Espasa, 2009.

Palomo Olmos, Bienvenido. "El afijo -ísimo en el español actual". Verba 28 (2001): 159-85.

Pastor Blanco, José María. "Caracteres lingüísticos de La Rioja (I): Claves fónicas y claves morfosintácticas”. Berceo 146 (2004): 7-65.

Pato, Enrique, y Julio Viejo. "Mucho bien y mucho guapa: mu(n)cho como cuantificador 'pleno' de adjetivos y adverbios en castellano y en asturiano". Bulletin of Spanish Studies 92.2 (2015): 1-18.

Pinkster, Harm. Latin Syntax and Semantics. Londres: Routledge, 1990.

Pons Rodríguez, Lola. "La doble graduación muy -ísimo". Estudios de Filología y Lingüística españolas: nuevas voces en la disciplina. Ed. Enrique Pato. Berna: Peter Lang, 2012. 135-66.

Rohlfs, Gerhard. Grammatica storica della lingua italiana e dei suoi dialetti. Turín: Einaudi, 1968. 
Sáez del Álamo, Luis Ángel. "Los cuantificadores: las construcciones comparativas y superlativas". Gramática descriptiva de la lengua española. Dirs. Ignacio Bosque y Violeta Demonte. Madrid: Espasa-Calpe, 1999. 1129-88. Sánchez López, Cristina. "Los cuantificadores: clases de cuantificadores y estructuras cuantificativas". Gramática descriptiva de la lengua española. Dirs. Ignacio Bosque y Violeta Demonte. Madrid: Espasa-Calpe, 1999. 1025128.

Sánchez López, Cristina. El grado de adjetivos y adverbios. Madrid: Arco Libros, 2006.

Serradilla Castaño, Ana. "Superlativos cultos y populares en el español clásico". Edad de Oro 23 (2004): 95-134.

Serradilla Castaño, Ana. "Evolución de la expresión del grado superlativo absoluto en el adjetivo: las perífrasis sustitutivas del superlativo sintético en español antiguo". CAUCE. Revista Internacional de Filología y su Didáctica 28 (2005): 357-85.

Serradilla Castaño, Ana. "El proceso de gramaticalización en las perífrasis de superlativo absoluto". Actas del VI Congreso Internacional de Historia de la Lengua española. Eds. José Luis Girón y José Jesús de Bustos. Madrid: Arco Libros, 2006. 1123-34.

Serradilla Castaño, Ana. "Bien + adjetivo como perífrasis de superlativo en español: particularidades semánticas y sintácticas”. Verba 33 (2007): 21533.

Serradilla Castaño, Ana. "Una diferencia sociolingüística en el uso de las fórmulas superlativas en español medieval". Discurso y sociedad, II. Nuevas contribuciones al estudio de la lengua en contexto social. Eds. José Luis Blas Arroyo y otros. Castellón: Publicacions de la Universitat Jaume I, 2008. 597-609.

Serradilla Castaño, Ana. "Los adjetivos relacionales desde una perspectiva diacrónica: características morfológicas, sintácticas y semánticas". Zeitschrift für Romanische Philologie 125.2 (2009): 197-242.

Sidwell, Keith. Reading Medieval Latin. Cambridge: Cambridge UP, 1995.

Wang, Chaofang. Las fórmulas superlativas en el español de los siglos XVIII y XIX. Madrid: Universidad Autónoma de Madrid, 2013.

Zieliński, Andrzej. "Evolución semántico-sintáctica del sufijo superlativo -ísimo en castellano”. Romanica Cracoviensa 13 (2013): 105-15. 